\title{
Distress Assessment and Response Systems in Patients With Cancer Pain
}

\author{
Hong Wei Li, Guang Yang, Xiao Hui Ma, Dong Sheng Xu, Ling Ling Zhao, Jiu Wei Cui, Nan Ya Wang
}

Cancer Center, the First Hospital of Jilin University, Changchun 130021, China

\begin{abstract}
Objective This study used the Distress Assessment and Response Tool (DART) to screen, evaluate, and explore physical symptoms, psychological problems, and social support in patients with cancer pain, with an aim to provide data for the comprehensive care of these patients to improve their quality of life. Methods The DART was used to survey 497 patients with cancer pain who were admitted to the Cancer Center of the First Hospital of Jilin University from June 2014 to March 2017. All patients were diagnosed with malignant tumors by clinical, radiological and/or cytological examinations, and were experiencing tumor-related pain. This study recorded the patient's somatic symptoms, psychological problems and social support in detail. After the data were collected, the SPSS 21.0 software was used to perform the statistical analysis, with the significance level set at $P<0.05$. Results In total, $82.7 \%$ of patients were experiencing mild pain (NRS $\leq 3$ ); of these, $29.7 \%$ and $15.8 \%$ reported depression and anxiety, respectively. In addition, $17.3 \%$ of patients were experiencing moderate or severe pain (NRS $\geq 4$ ); of these, $53.5 \%$ and $40.7 \%$ reported depression and anxiety, respectively. There was a statistically significant difference in the incidence of anxiety and depression between the two groups. Conclusion Patients with cancer pain, particularly those with moderate-to-severe pain, showed a high incidence of distress. In addition, they were likely to report physical symptoms, were conscious of poor health conditions, and were prone to have anxiety and depression. In terms of social support, patients with moderate-to-severe cancer pain should receive substantial support, as they experience work-related and economic concerns and report difficulties in family life and social activities.
\end{abstract}

Key words: Cancer pain; Distress; Pain assessment; Distress Assessment and Response Tool (DART); Anxiety

\section{Introduction}

Pain is one of the most common and frightening symptoms of cancer, and it has been estimated that $75 \%$ to $90 \%$ of patients with metastatic and advanced cancer experience pain [1]. Cancer pain leads to serious negative effects on the patients'diet, sleep, activity and causes negative emotions, such as anxiety, depression, and frustration, which increase the patients' suffering, affects treatment, and leads to a poorer quality of life [2]. Therefore, the National Comprehensive Oncology Network and American Society of Clinical Oncology stated that cancer patients should be screened for distress and their psychological problems should be identified as soon as possible in order to implement appropriate intervention measures [3]. Since the application of the Distress Thermometer (DT) in China in 2007, clinical staff have significantly increased the clinical screening for and research related to patients' pain. In 2016, the Chinese Psychosocial Oncology society (CPOS) published the 1st practice guidelines regarding the psychosocial intervention for cancer patients, which highlighted the importance of incorporating pain screening into routine cancer treatment. However, there have been few studies on the results of such

Hong Wei Li and Guang Yang are contributed equally to this work. Corresponding author: Professor Nan Ya Wang, PhD, Cancer Center, The First Hospital of Jilin University, Xinmin Street 71\#, Jilin, Changchun, 130021 China; Tel: +86 1580430 2611; Fax: +86 0431 8878 3373; Email: nanyawang@yahoo.com screening for cancer patients' distress in China, and the sample sizes were small. There were also differences in the structures and nature of the assessment tools used, with no established standard.

The Distress Assessment and Response Tool (DART) is a pain screening questionnaire commonly used in many hospitals in the U.S. and Canada. The questionnaire has been clinically proven to be easy to use and can be applied in large-scale studies and for the routine screening of cancer patients. In addition, it is suitable for use in the early detection of patients' psychological distress, which could help medical staff to intervene, relieve their distress, and improve the outcomes of treatment [4]. This study aimed to determine the extent of distress in patients with cancer pain to provide evidence to inform the development of appropriate interventions, as well as to establish a referral mechanism for psychological and social support, and to promote the development of multidisciplinary therapy.

\section{Materials and methods Participants}

We used convenience sampling to recruit 497 patients with cancer pain who had been screened for distress at the Cancer Center at the First Hospital of Jilin University between June 2014 and March 2017. In total, 500 questionnaires were distributed, of which 497 (99.4\%) were valid. Patients with cancer pain were divided into two groups ac- 
cording to the extent of their pain: a mild cancer pain group (NRS $\leq 3$ points) and a moderate-to-severe cancer pain group (NRS $\geq 4$ points).

The inclusion criteria were

(1) A pathological or clinical diagnosis of malignancy,

(2) Pain,

(3) Knowledge of the disease condition and sufficient consciousness to complete the questionnaire

\section{Measurement instruments}

The DART was used to evaluate the patients' symptoms and concerns. It includes two sections: the first collects data regarding basic information, including the sex, age, diagnosis, and marital status, while the second assesses the existing symptom burden, anxiety, depression, activity, social support, and work-related and economic concerns. Symptom screening was performed using the Edmonton Symptom Rating Scale, which includes items pertaining to pain, fatigue, nausea, sleep, appetite, well-being, and dyspnea. The severity of each symptom is rated using a scale ranging from 0 to 10, where a score of 0 indicates asymptomatic and a score of 10 indicates the most serious degree imaginable. Patients are asked to choose the number that best matches the severity of their average feelings. The pain severity was classified as follows: 1 3: mild pain; 4 6: moderate pain; and 7 10: severe pain, where scores of $\geq 4$ indicate that the patient is always experiencing pain. The severity of other symptoms was graded using the same scale with reference to a previous study [5]: 1 3: mild; 4 6: moderate; and 7 10: severe. Patients with anxiety and depression were also assessed using the Edmonton Symptom Rating Scale (ESAS-A, ESAS-D). Depression scores $\geq 2$ were classified as indicating depression, anxiety scores $\geq 3$ were classified as indicating anxiety.

\section{Procedure}

The researchers received unified training about how to explain the questionnaire to patients and obtain consent. The patients completed the questionnaire at the hospital.

\section{Statistical analysis}

The data were processed using the SPSS 21.0 software. The counted data were expressed as frequencies and percentages $(\%)$. A chi-squared test was performed to compare two groups. The significance level was set at $P<$ 0.05 .

\section{Results \\ Patient characteristics}

Of the 497 patients, 243 were men and 254 were women (median age: 56 years). Of these patients, 186, 104, 90, 55, 28,28 , and 6 had lung cancer, gastrointestinal tumors, breast cancer, liver cancers, gynecological tumors, malignant hematological disease, and osteosarcoma, respectively. In addition, 411 patients experienced mild cancer pain, while 86 experienced moderate-to-severe cancer pain.

\section{Symptom burden in cancer patients}

The symptom burden in patients with cancer pain is shown in Table 1. In the mild cancer pain group, the symptoms included loss of appetite (30.9\%), fatigue (29.4\%), dyspnea (17\%), sleep disorders (15.3\%), and nausea $(13.9 \%)$. In the moderate-to-severe cancer pain group, the symptoms included fatigue (74.4\%), loss of appetite (68.6\%), nausea (48.8\%), sleep disorders (47.7\%), and dyspnea $(47.7 \%)$. Further, $35.3 \%$ and $76.7 \%$ of patients in the mild and moderate-to-severe cancer pain groups respectively reported having generally poor well-being.

\section{Anxiety and depression in patients with cancer pain}

The incidence rates for anxiety and depression in the mild cancer pain group were $15.8 \%$ and $29.7 \%$, respectively. In contrast, the rates were $40.7 \%$ and $53.5 \%$, respectively, in the moderate-to-severe cancer pain group. The incidence of depression was significantly higher $(P<0.05)$ than that of anxiety in both groups. The data are shown in Table 2.

\section{Problems affecting patients with cancer pain and self-re- ported depression}

Of 497 patients with cancer pain, 168 (33.8\%), including $122(24.5 \%)$ in the mild cancer pain group and 46 (9.3\%) in the moderate-to-severe cancer pain group, reported experiencing depression. The problems that had affected patients during the preceding 2 weeks included sleep disorders $(86.3 \%)$, a lack of interest in normal activities $(85.1 \%)$, fatigue $(82.7 \%)$, low spirits $(81.5 \%)$, loss of appetite $(66.7 \%)$,

Table 1 The Patients' Scores on the Edmonton Symptom Rating Scale (n, \%).

\begin{tabular}{|c|c|c|c|c|c|c|}
\hline \multirow[t]{2}{*}{ Symptoms } & \multicolumn{3}{|c|}{ Mild cancer pain } & \multicolumn{3}{|c|}{ Moderate-to-severe cancer pain } \\
\hline & Mild & Moderate & Severe & Mild & Moderate & Severe \\
\hline Fatigue & $290(70.6)$ & $97(23.6)$ & $24(5.8)$ & $22(25.6)$ & $41(47.7)$ & $23(26.7)$ \\
\hline Nausea & $354(86.1)$ & $44(10.7)$ & $13(3.2)$ & $44(51.2)$ & $29(33.7)$ & $13(15.1)$ \\
\hline Sleep disorders & $348(84.7)$ & 49 (11.9) & $14(3.4)$ & $45(52.3)$ & $27(31.4)$ & $14(16.3)$ \\
\hline Loss of appetite & $284(69.1)$ & $90(21.9)$ & $37(9.0)$ & $27(31.4)$ & $39(45.3)$ & $20(23.3)$ \\
\hline Poor well-being & $266(64.7)$ & $114(27.7)$ & $31(7.6)$ & $20(23.3)$ & $41(47.6)$ & $25(29.1)$ \\
\hline Dyspnea & $341(83.0)$ & $55(13.4)$ & $15(3.6)$ & $45(52.3)$ & $23(26.7)$ & $18(21.0)$ \\
\hline
\end{tabular}


difficulty concentrating (60.7\%), feeling disappointed in oneself or family (48.8\%), irritability $(41.7 \%)$, and the impulse to engage in self-harm (35.7\%), as shown in Table 2. Significant differences in fatigue, loss of appetite, and irritability were observed between the patients with mild versus moderate-to-severe pain $(P<0.05)$.

\section{Problems affecting patients with cancer pain and self-re- ported anxiety}

Of the 497 patients with cancer pain, 100 (20.1\%), including $65(13.1 \%)$ in the mild pain group and $35(7.0 \%)$ in the moderate-to-severe cancer pain group, reported anxiety. Problems that had affected patients within the preceding 2 weeks included tension (87\%), excessive worry $(81 \%)$, difficulty relaxing $(73 \%)$, irritability (73\%), fidgeting $(66 \%)$, uncontrolled fear (57\%), and fear of impending doom (57\%), as shown in Table 3. There were no significant differences in these problems between the two groups.

\section{Social support}

The results of the analysis of social support are shown in Table 4. Social support includes substantial support, concerns about work and economic, family life and social activities. There were statistically significant differences in the areas where support was needed between the mild and moderate-to-severe pain groups $(P<0.05)$. The results showed that a small number of patients in both groups experienced suicidal ideation, and most patients were unwilling to receive supportive services (Table 4). There were no significant differences between the two groups in terms of suicidal ideation or the willingness to receive support.

\section{Discussion}

Malignant tumors often have high recurrence rates, endanger patients' lives, create a heavy symptom burden, and cause patients great physical and psychological distress. The cancer pain treatment can effectively reduce the pain level and the incidence of adverse reactions, as well as improve the physical condition and quality of life of patients with terminal cancer. Therefore, psychological distress in patients with cancer requires the attention of medical staff. Studies conducted abroad have demonstrated a high rate of pain when the DART was used as a screening tool in cancer patients [6]. A cross-sectional study conducted by

Table 2 Self-reported anxiety and depression affecting patients with cancer pain (n, \%).

\begin{tabular}{|c|c|c|c|c|c|}
\hline & \multicolumn{2}{|c|}{ Mild cancer pain } & \multicolumn{2}{|c|}{ Moderate-to-severe cancer pain } & \multirow[t]{2}{*}{$P$} \\
\hline & without & with & without & with & \\
\hline \multicolumn{6}{|l|}{ Self-reported conditions } \\
\hline Depression & $289(70.3)$ & $122(29.7)$ & $65(15.8)$ & $46(53.5)$ & $<0.001$ \\
\hline Anxiety & $346(84.2)$ & $65(15.8)$ & $51(59.3)$ & $35(40.7)$ & $<0.001$ \\
\hline \multicolumn{6}{|l|}{ Individual symptoms of depression } \\
\hline Lack of interest in normal activities & $67(16.4)$ & $343(83.6)$ & $9(10.9)$ & $77(89.1)$ & 0.370 \\
\hline Low spirits & $84(20.5)$ & $326(79.5)$ & $11(13.0)$ & $75(87.0)$ & 0.267 \\
\hline Sleep disorders & $57(13.9)$ & $354(86.1)$ & $11(13.0)$ & $75(87.0)$ & 0.881 \\
\hline Fatigue & $91(22.1)$ & $320(77.9)$ & $4(4.3)$ & $82(95.7)$ & 0.007 \\
\hline Loss of appetite & $155(37.7)$ & $256(62.3)$ & $19(21.7)$ & $67(78.3)$ & 0.050 \\
\hline Disappointment in oneself or family & $216(52.5)$ & $195(47.5)$ & $41(47.8)$ & $45(52.2)$ & 0.592 \\
\hline Difficulty concentrating & $165(40.2)$ & $246(59.8)$ & $32(37.0)$ & $54(63.0)$ & 0.704 \\
\hline Irritability & $259(63.1)$ & $152(36.9)$ & $39(45.7)$ & $47(54.3)$ & 0.041 \\
\hline Impulse to engage in self-harm & $273(66.4)$ & $138(33.6)$ & $50(58.7)$ & $36(41.3)$ & 0.353 \\
\hline
\end{tabular}

Table 3 Problems affecting patients with cancer pain and self-reported anxiety (n, \%).

\begin{tabular}{|c|c|c|c|c|c|}
\hline \multirow[t]{2}{*}{ Problems } & \multicolumn{2}{|c|}{ Mild cancer pain } & \multicolumn{2}{|c|}{ Moderate-to-severe cancer pain } & \multirow[t]{2}{*}{$\boldsymbol{P}$} \\
\hline & without & with & without & with & \\
\hline Tension & $44(10.8)$ & $367(89.2)$ & $15(17.1)$ & $71(82.9)$ & 0.366 \\
\hline Uncontrolled fear & $177(43.1)$ & $234(56.9)$ & $37(42.9)$ & $49(57.1)$ & 0.983 \\
\hline Excessive worry & $88(21.5)$ & $322(78.5)$ & $12(14.3)$ & $74(85.7)$ & 0.378 \\
\hline Difficulty relaxing & $127(30.8)$ & $284(69.2)$ & $17(20.0)$ & $69(80.0)$ & 0.247 \\
\hline Fidgeting & $139(33.8)$ & $272(66.2)$ & $29(34.3)$ & $57(65.7)$ & 0.965 \\
\hline Irritability & $127(30.8)$ & $284(69.2)$ & $17(20.0)$ & $69(80.0)$ & 0.247 \\
\hline Fear of impending doom & $177(43.1)$ & $233(56.9)$ & $37(42.9)$ & $49(57.1)$ & 0.983 \\
\hline
\end{tabular}


Alemayehu M et al. [7] showed that the prevalence of moderate-to-severe depression in 390 cancer patients was $16.4 \%$ (95\% CI: 13.1-20.4). In the current study, the incidence rates for self-reported depression and anxiety were $33.8 \%$ and $20.1 \%$, respectively, in patients with moderate-to-severe pain. Because of regional cultural differences, where Chinese residents are often unwilling to talk to others about their pain, the results of the current study differed from those of studies conducted in other countries.

The results of the current study showed that patients with cancer pain experienced one or more physical symptoms such as fatigue, nausea, and loss of appetite. The incidence of coexistent symptoms in the moderate-to-severe cancer pain group was higher, and the severity of these symptoms was greater than that observed in the mild cancer pain group. These findings were consistent with the results of the overall status of well-being, where $35.3 \%$ and $76.7 \%$ of patients in the mild and moderate-to-severe cancer pain groups respectively reported poor overall well-being. The occurrence of physical symptoms, such as fatigue, nausea, sleep disorders, and appetite loss, decrease the patients' quality of life, causing them to worry about their health, leading to anxiety, depression, despair, and other negative emotions and further physical and psychological symptoms and pain. It has been shown that patients' psychological responses evolve from a single symptom to a complex syndrome [8]. Chen J et al. [9] studied the psychological pain of breast cancer patients and showed that while the factors that caused psychological pain varied, their interaction was obvious, and most pain was considered to be due to a comprehensive set of factors. Zheng $\mathrm{H}$ et al. suggested that the anxiety and depression of patients with cancer pain are significantly positively correlated with the degree of pain, where a higher degree of pain was associated with more obvious anxiety and depression [10]. Laird BJA et al. found that pain and depression are both common in cancer patients [11]. A different study by Anderson $\mathrm{KO}$ et al. showed that $67 \%$ of patients' pain was associated with the psychological pain caused by cancer; $33 \%$ said that the pain was sometimes unbearable and they wanted to die; $69 \%$ of patients had difficulty in daily activities because of pain; $41 \%$ of the patients indicated that their pain affected their relationship with their family [12].
In both groups in the current study, the incidence of depression was higher relative to that of anxiety. In addition, there was a significant difference in the incidence of anxiety and depression between the two groups $(P<0.05)$. Not surprisingly, the results showed that the incidence of both anxiety and depression was higher and the severity of symptoms was greater in patients with moderate-to-severe cancer pain relative to those in patients with mild pain. These results are consistent with the results reported by Zhu Y et al. [13]. The present study shows that the incidence of depression and anxiety increased with the incidence of pain. The incidence of depression and anxiety in patients with mild cancer pain was $10 \% \sim 20 \%$, while the incidence in patients with moderate cancer pain was $70 \% \sim 80 \%$, and the incidence in patients with severe cancer pain was higher than $85 \%$, so the incidence of depression and anxiety in patients with moderate or severe cancer pain was higher than that in patients with mild cancer pain $(P>0.05)$. Therefore, medical staff should pay particular attention to the psychological distress of patients with moderate-to-severe cancer pain.

There was no statistically significant difference between the two groups with regard to the problems that affected patients with cancer pain and anxiety. However, while depression affected most patients with cancer pain, there were statistically significant differences between the two groups in the incidence of fatigue, loss of appetite, and irritability $(P<0.05)$. The results showed that the physical symptoms reported by patients with moderate-to-severe cancer pain were likely to decrease the positive emotions and quality of life of the patients, and to increase the negative emotions of the patients. Therefore, medical staff should provide clinical interventions for patients with cancer pain to alleviate symptoms. Such interventions will require screening for problems that are causing patients distress.

There was a significant difference in social support between the two groups $(P<0.05)$. Significantly more patients in the moderate-to-severe cancer pain group accepted substantial support in areas including daily activities, travel, and personal care; they also reported more concerns about work, family, economic issues, and social interactions compared to the patients with mild cancer pain. Although there were concerns about social support in both groups, the patients did not express high levels of willingness to

Table 4 Social support and patients' willingness to receive support (n, \%).

\begin{tabular}{|c|c|c|c|c|c|}
\hline & \multicolumn{2}{|c|}{ Mild cancer pain } & \multicolumn{2}{|c|}{ Moderate-to-severe cancer pain } & \multirow[t]{2}{*}{$P$} \\
\hline & without & with & without & with & \\
\hline \multicolumn{6}{|l|}{ Areas where support is needed } \\
\hline Substantial support needed every day & $269(65.5)$ & $142(34.5)$ & $41(47.7)$ & $45(52.3)$ & 0.002 \\
\hline Work-related and economic concerns & $211(51.3)$ & $200(48.7)$ & $32(37.2)$ & $54(62.8)$ & 0.017 \\
\hline Concerns about family life and social activities & $328(79.8)$ & $83(20.2)$ & $60(69.8)$ & $26(30.2)$ & 0.041 \\
\hline Suicidal ideation & $364(88.5)$ & $47(11.5)$ & $79(91.3)$ & $7(8.7)$ & 0.603 \\
\hline Willing to receive support services & $304(74.0)$ & $107(26.0)$ & $56(65.1)$ & $30(34.9)$ & 0.095 \\
\hline
\end{tabular}


accept supportive services; this could have been related to the cultural background in China. Zhang GF et al. found that the psychological pain in patients with cancer pain is closely related to anxiety, depression and the level of social support. Interventions should include psychological counseling, mental support and social support for patients with cancer pain to improve their psychological state and eliminate barriers to receiving support, thereby improving the patient's quality of life [14]. Howell D et al. found that cognitive behavioral interventions effectively improved insomnia in adult cancer patients [15]. Kuchler $\mathrm{T}$ et al. found that psychological support can benefit the survival of gastrointestinal cancer patients [16]. These studies highlight the importance of psychosocial support.

The results of the current study showed that the distress experienced by patients with cancer pain was related to fatigue, sleep disorders, appetite loss, mobility loss, and negative emotions. Patients with moderate-to-severe cancer pain showed greater decreases in quality of life relative to those in patients with mild cancer pain. This was likely because they have more difficulties in work, family life and social activities, and need more substantive support. Medical staff should provide targeted clinical interventions for patients with cancer pain to alleviate their suffering, especially for patients with moderate-to-severe cancer pain. It is believed that with improved awareness of these psychological effects of pain and the introduction of treatments, these patients will experience an improved quality of life. Domestic research has shown that the severity of distress is strongly positively correlated with physical and emotional problems, as well as issues with social interactions [17]. Chen L et al. examined psychological distress in patients with lung cancer, and found that poor control of somatic pain was a risk factor for psychological distress. Furthermore, mental pain could exacerbate somatic pain to form a vicious cycle [18]. Keefe FJ et al. [19] showed that there is a relationship between pain and psychological factors, wherein psychological pain aggravates the patient's physical pain and hinders the effective management of pain. Liu $\mathrm{H}$ et al. suggested that the implementation of the standardized treatment of cancer pain can not only alleviate the pain of patients with advanced cancer, but can also reduce the incidence of adverse drug reactions, and improve the quality of life [20]. Therefore, it is necessary to strengthen the management of patient pain. Medical staff must not only recognize the degree of pain and the nature of the pain, but also pay attention to the interference of the pain with various aspects of daily life, thereby reducing the degree of psychological pain of patients.

During anti-tumor therapy, patients generally experience one or more (frequently coexistent) symptoms such as pain, nausea, and fatigue, with a higher number of symptoms related to a greater likelihood of anxiety and depression. Because of the impact of cultural beliefs in China, patients are reluctant to express their negative emotions, which leads to difficulties for the medical staff in assessing patients and in- tervening to alleviate the patients' distress. Cancer treatment is a long and difficult process during which the patient's psychological health is affected by various factors such as the physical suffering, family relationships, and interactions with society, including work. Therefore, quick and effective assessment of the extent of a patient's psychological distress and targeted interventions to alleviate physical and psychological problems could effectively reduce the incidence of anxiety and depression, relieve patients' psychological distress, encourage the patient to accept support, and improve their quality of life.

\section{Conclusion}

Patients with cancer pain, particularly those with moderate-to-severe pain, showed a high incidence of distress. In addition, they were likely to report physical symptoms, were conscious of poor health conditions, and were prone to have anxiety and depression. In terms of social support, patients with moderate-to-severe cancer pain should receive substantial support, as they experience work-related and economic concerns and report difficulties in family life and social activities.

\section{Conflict of Interests}

The authors declared that they have no conflicts of interest related to this work.

\section{Funding}

This work was supported by Jilin University Excellent Young Teacher Training Program (No. 419080500356)

\section{References}

1. Falk S, Dickenson A H. Pain and nociception: mechanisms of cancer-induced bone pain. J Clin Oncol 2014;32(16):1647-54.

2. Grassi L, Johansen C, Annunziata MA, Capovilla E, Costantini

A, Gritti P, Torta R, Bellani M; Italian Society of Psycho-Oncology Distress Thermometer Study Group.Screening for distress in cancer patients: a multicenter, nationwide study in Italy. Cancer 2013;119(9): 1714-21.

3. Faller H, Schuler M, Richard M, Heckl U, Weis J, Küffner R. Effects of psycho-oncologic interventions on emotional distress and quality of life in adult patients with cancer: systematic review and meta-analysis. J Clin Oncol 2013;31(6):782-93.

4. Li M, Macedo A, Crawford S, Bagha S, Leung YW, Zimmermann C, Fitzgerald B, Wyatt M, Stuart-McEwan T, Rodin G. Easier said than done: Keys to successful implementation of the Distress Assessment and Response Tool (DART) Program. J Oncol Pract 2016 May;12(5):e513-26.

5. Selby D, Cascella A, Gardiner K, et al. A single set of numerical cutpoints to define moderate and severe symptoms for the edmonton symptom assessment system. J Pain Symptom Manage 2010 Feb;39(2):241-9.

6. Bagha S M, Macedo A, Jacks L M, Lo C, Zimmermann C, Rodin G, Li M. The utility of the Edmonton Symptom Assessment System in screening for anxiety and depression. Eur J Cancer Care (Engl) 2013 Jan;22(1):60-9. 
7. Alemayehu, M, Deyessa N, Medihin G, Fekadu A. A descriptive analysis of depression and pain complaints among patients with cancer in a low income country. PLoS One 2018 Mar 7;13(3):e0193713.

8. Li L, Chen J, Liang W. Research progress on assessment and intervention for psychological distress of cancer patients. Chin Nur Res 2015(28): 3462-4

9. Chen J, Liu J, Su Y, Wang H. Psychological distress and its relevant factors among inpatients with breast cancer. J Nurs Adm 2012;12(3): 158-61.

10. Zheng H, Zhang N, Cai YY, Effects of psychological intervention on cancer pain, anxiety and depression in cancer patients. Oncol Pro 2017;15(10):1229-31.

11. Laird BJA, Boyd AC, Colvin LA, Fallon MT. Are cancer pain and depression interdependent? A systematic review. Psycho-Oncology 2009; 18(5):459-64.

12. Anderson KO, Green CR, Payne R. Racial and ethnic disparities in pain: causes and consequences of unequal care. J Pain 2009;10:1187204.

13. Zhu Y, Zhu JQ, Cai GY. Investigation on anxiety and depression of patients with different degrees of cancer pain. Chin Prev Med 2016(6):455-7.

14. Zhang GF, Zhang Y. Analysis of psychological pain level and influencing factors in patients with cancer pain. Global Tradit. Chin Med
2013;6(S2):10-1.

15. Howell D, Oliver TK, Keller-Olaman S, Davidson J, Garland S, Samuels C, Savard J, Harris C, Aubin M, Olson K, Sussman J, Macfarlane J, Taylor C. Sleep Disturbance Expert Panel on behalf of the Cancer Journey Advisory Group of the Canadian Partnership Against Cancer. A Pan-Canadian practice guideline: prevention, screening, assessment, and treatment of sleep disturbances in adults with cancer. 2013;21(10):2695-706.

16. Küchler T, Bestmann B, Rappat S, Henne-Bruns D, Wood-Dauphinee S. Impact of psychotherapeutic support for patients With gastrointestinal cancer undergoing surgery: 10-year survival results of a randomized trail. J Clin Oncol 2007;25(19):2702-8.

17. Li C, Zhang $X$, Liu Z. Investigation of psychological pain in patients with bladder cancer. Shaanxi Yixue Zazhi 2014(10):1424-5.

18. Chen L, Zheng R, Yu C, Li J. Impact factors of distress in patients with lung cancer-associated pain. Sichuan Da Xue Xue Bao Yi Xue Ban 2014;45(3):471-5.

19. Keefe FJ, Rumble ME, Scipio CD, Giordano LA, Perri LM. Psychological aspects of persistent pain: current state of the science. J Pain 2004;5(4):195-211.

20. Liu H, Jiang YJ, Liu SX. Effcacy of standardized cancer pain treatment for improving quality of life in patients with severe cancer pain. Acad J Third Mil Med Univ 2018;40(10):934-8. 